by increase of cerebral blood flow. The intracranial pressure decreased in all cases. EEG revealed slight improvement. The diameter of cortical and peripheral vessels did not change during the experimental period. These results suggested that the administration of mannitol brought about a beneficial effect on cerebral edema.

\title{
S-B-8. Effect of Hyperbaric Oxygenation on Cerebral Edema
}

\author{
Dennosuke Jinnai, Heitaro Mogami, Masahiko IokU, Kiyoo Kamikawa, \\ Jiro Mukawa, Takuya IKeda, Kentaro Koshino, Toru HayaKawa, \\ Ryotaro Kuroda, Kikushi Katsurada, Nobuhiro KanaI, Ryohei Yamada, \\ Yoshikazu Iwata, Hiromasa Murui, Masaharu HorI, Michio Ogawa, \\ Kiyofumi Kobayashi, Mamoru Taneda and Ichiro Tahara \\ 2nd Department of Surgery, Osaka University Medical School
}

In previous studies, the cerebral edema associated with stereotaxic local cooling and chemotherapy of brain tumor has been reported by our laboratory. This study was undertaken to evaluate the effectiveness of hyperbaric oxygenation (OHP) on clinical and experimental cerebral edema.

In our clinic, 15 cases suffering from severe neurologic disorders (traumatic: 7 cases, postoperative: 8 cases) were treated with OHP. Some degree of clinical neurological improvements were noticed in most cases and improvements of EEC were also seen in some of them during the exposure to OHP ( 2 or 3 ATA). However, the most of the improvements were temporary and the regrssions occurred after decompression. Moreover, in a few cases, the progressive downhill courses were seen after the treatments.

Improvements of EEG during the exposure to OHP were obtained in cats subjected to cerebral edema produced by extradural balloon technique. Another experiment was designed to study the protective effect of OHP on cerebral edema produced by internal carotid oil infusion technique in rabbits. All control animals which were not treated with OHP died within one hour after oil infusion of $0.03 \mathrm{ml}$ per $\mathrm{kg}$, but a few of the animals treated with OHP ( 3 ATA for one hour immediately after infusion) survived until they were killed 3.5 hours later. However, with oil infusion of $0.015 \mathrm{ml}$ per $\mathrm{kg}$, some of the OHP treated animals died after decompression, though none of the controls died. In addition to these, the influ. ence of OHP on the blood brain barrier was studied after internal carotid mitomycin $\mathrm{C}$ infusion $(1 \mathrm{mg} / \mathrm{kg})$ in rabbits. The destruction of the blood brain barrier was estimated by measuring the uptake ratio of intravenously injected RISA in the cerebral tissue. Unexpectedly, the higher RISA uptake was observed in the OHP 
treated group ( 3 ATA for one hour, 3 times every 8 hours) than the control group. The influence of OHP on CSF pressure was studied. The CSF pressure decreased by increment of oxygen pressure in hyperbaric chamber and returned to the former level after decompression. However, the exposure to OHP for a long time resulted in gradually increasing CSF pressure, which had decreased at initial stage of OHP and, after decompression, amounted to the higher level than the level at pretreatment. Such a rebound phenomena of CSF pressure inclined to occur in the animals, in which $\mathrm{PaCO}_{2}$ decreased during OHP. It was presumed that the lowering of CSF pressure during the exposure to OHP was due to cerebral vasoconstriction. On the basis of clinical and experimental findings, it can be postulated that the rebound phenomena of CSF pressure is caused by the destruction of the blood brain barrier in addition to cerebral vasodilatation. The next attempt was made to counteract cerebral vasoconstriction which decreases cerebral blood flow and herefore a quantity of carbon dioxide was mixed in breathing oxygen. Measuring CSF $\mathrm{PO}_{2}$ as an indicator related to cerebral oxygen content, the addition of $2 \%$ $\mathrm{CO}_{2}$ to ventilating oxygen significantly increased $\mathrm{CSF} \mathrm{PO}_{2}$ in comparison with the one in the breathing pure oxygen and this finding suggested the increment of :erebral blood flow. However, when the patients with neurologic disorders reathed $98 \% \mathrm{O}_{2}$ plus $2 \% \mathrm{CO}_{2}$ mixture under $2 \mathrm{ATA}$, the CSF pressure increased narkedly and the neurologic deterioration was found. This result suggests that ess $\mathrm{CO}_{2}$ than $2 \%$ may be suitable for the patients with severe neurologic disorders $\supset$ breath. As far as the rebound phenomena is concerned, it can be noted that the shenomena should be prevented if $\mathrm{PaCO}_{2}$ level maintains somewhat higher than $.0 \mathrm{mmHg}$ in dogs. Histopathologically, the evidence of effect on OHP treatment or cerebral edema has not yet been found.

It may be concluded that OHP treatment improves neurologic disorders ffected by cerebral edema-improvements of clinical behaviors and EEG findings -, but on the contrary, it is apt to damage the blood brain barrier and thus inreases cerebral edema. Further studies on prophylactic prevention of oxygen xity, counteraction of cerebral vasoconstriction, decompression schedule for the atients with severe cerebral damages, combination with other treatments for rebral edema, etc.-are necessary for clinical use of OHP treatment. 